\title{
Spatial-temporal Variation of Soil Moisture in China from Long Time Series Based on GLDAS-Noah
}

\author{
Mengqing Geng, Feng Zhang, ${ }^{*}$ Xiaoyan Chang, ${ }^{* *}$ Qiulan Wu, and Lin Liang \\ Shandong Agricultural University, Tai'an, 271000, China
}

(Received May 28, 2021; accepted November 11, 2021; online published December 13, 2021)

Keywords: China, soil moisture, spatial-temporal variability, GLDAS, Mann-Kendall, Hurst

Soil moisture is a comprehensive reflection of soil moisture status and is an important parameter for land surface conditions. It is very important to study the distribution characteristics of soil moisture for ecological environment protection, scientific and rational utilization of soil water resources, and climate research. Using the soil layer humidity data sets of GLDAS-Noah v2.0 and v2.1, we analyzed the spatial-temporal distribution of soil moisture in China in a layer from 0 to $200 \mathrm{~cm}$ over 71 years from 1948 to 2018. Firstly, the Mann-Kendall trend test was used to analyze the trend of the changes and the spatial variation characteristics of soil moisture over the 71 years. Secondly, the coefficient of variation was used to analyze the temporal and spatial fluctuation of soil moisture in each layer of the study area over the 71 years. Finally, the Hurst index was used to predict the future trend of soil moisture changes in each layer. In addition, the correlation between soil moisture and the spatial-temporal variation of soil temperature in China was explored. The results show that the annual variation trend of soil moisture in the $0-200 \mathrm{~cm}$ soil layer has been consistent, that is, the soil humidity in most parts of east China has been decreasing, especially in northeast China, central China, the area surrounding the Yunnan Guizhou Plateau, and Taiwan Island, while it has been increasing in most of the western regions. Also, the change in soil layer humidity from 0 to $200 \mathrm{~cm}$ in southern China was greater than that in the northern region, and the humidity of the soil layer in the Pearl River Delta region was the most unstable. In addition, the spatial variation of soil moisture in the study area was relatively small from 1948 to 2001, but from 2002, the soil moisture throughout the study area became uneven. In the future, the trend of the change in soil moisture in most areas of China will remain consistent with that in the past 71 years, i.e., the soil in most parts of the east will gradually dry out and the soil moisture in most parts of the west will gradually increase; the soil humidity from 0 to $200 \mathrm{~cm}$ in most of the study area is inversely related to the soil temperature, and is mainly concentrated in northeast and central China, central and northern Inner Mongolia, the Qinghai Tibet Plateau, and Taiwan Island.

\section{Introduction}

Soil moisture is the material basis for plant growth and survival, and it is also a medium for material and energy exchange among soil, plants, and the surrounding environment. Therefore,

\footnotetext{
*Corresponding author: e-mail: zhangfeng@sdau.edu.cn

** Corresponding author: e-mail: xychang@sdau.edu.cn

https://doi.org/10.18494/SAM.2021.3445
} 
different soil moisture conditions lead to different ecosystem distributions. ${ }^{(1)}$ At the same time, a change in soil moisture will change the soil albedo and evapotranspiration and affect the soil heat capacity. These changes will affect the balance of the surface energy budget and eventually contribute to climate change. ${ }^{(2,3)}$ As an important physical quantity of the land surface, it is very important to fully understand the evolution process of its spatial-temporal pattern to control changes in soil moisture, protect the ecological environment, and make scientific and rational use of soil water resources for the study of climate and land surface processes.

Previous research has been carried out on soil moisture throughout China. For example, Zhang et al. ${ }^{(4)}$ analyzed the temporal and spatial distribution of soil moisture in a layer from 0 to $50 \mathrm{~cm}$ in 12 climatic regions of China using soil moisture data from 155 agricultural meteorological observation stations from 1981 to 2010. They used trend analysis and the Cramér-von Mises method to explore the trend in the changes in soil moisture from 1981 to 2010. Zhang et al. (2) $^{(2)}$ used soil moisture data obtained at depths of 0-100 cm from 1981 to 2000 in 57 stations in China to diagnose and analyze the soil moisture in the vertical direction from station to station. They classified the vertical distribution of humidity into three main types: summer uniform type, rapid change type, and seasonal difference type, and the inter-annual variation of soil humidity was analyzed. The above authors and others all obtained soil moisture data through site observation. These sites were relatively representative, for example, the stations were located in subtropical humid and semi-humid areas and temperate semi-humid, semi-arid, and arid climate areas, involving various types of soil such as black soil, desert soil, brown soil, and red soil. However, because of the limited number of research sites, some of the characteristics of the temporal and spatial variability of soil moisture in China may not have been revealed. Using National Centers for Environmental Prediction Climate Forecast System Reanalysis (NCEP-CFSR) data from 1981 to 2008, Zhang et al. ${ }^{(3)}$ divided China and its surrounding areas into three regions: south, north, and middle, studied the statistical properties of soil moisture in each region, compared and analyzed each region, and summarized the temporal and spatial distribution characteristics of summer precipitation and soil moisture. However, this method of dividing an area into regions in advance does not consider the internal heterogeneity of the regions, and whether this zoning method is reasonable and appropriate requires further discussion.

The lack of soil moisture observation data has seriously limited the development of soil climatology. At present, the widely used soil moisture data worldwide include reanalysis data, such as ERA-Interim, ERA40, and $\mathrm{NCEP}^{(5,6)}$ satellite remote sensing data, ${ }^{(7-9)}$ and assimilation data products, ${ }^{(10)}$ which make up for the serious shortage of observation data to a great extent. Sun et al. ${ }^{(11)}$ pointed out that the soil moisture data in GLDAS-Noah v2.0 has good applicability in the Qinghai Tibet Plateau, and the data set provides strong scientific support for the lack of spatial-temporal observation in the plateau. Kim et al. ${ }^{(12)}$ found that the average correlation coefficient between soil moisture from GLDAS-Noah and global in situ observations was 0.73 , which was higher than the values for ASCAT and AMSR2.

These previous studies have analyzed many temporal and spatial characteristics of soil moisture in China, but there are still some issues that need to be further explored: (1) the spatial variability of past long-term changes in soil moisture in China, (2) the spatial distribution of the 
time series coefficient of variation $\left(C_{v}\right)$ of soil moisture and the time fluctuation of the spatial distribution $C_{v}$ of soil moisture, (3) possible trends of soil moisture changes in China in the future, and (4) the relationship between soil moisture and soil temperature in China.

To address these issues, we studied the characteristics of the temporal and spatial variability of soil moisture in China from a new perspective. By considering the pixel of each soil layer as the basic unit, we studied the temporal and spatial distribution and variation characteristics of soil moisture in a $0-200 \mathrm{~cm}$ soil layer over 71 years as well as predicted future trends to find some spatial and temporal variation characteristics of soil moisture in China that have not been found before. The novel features of this study are as follows: (1) The Mann-Kendall (M-K) trend test was used to calculate the $Z$ value of each pixel to find the variation trends of soil moisture in a $0-200 \mathrm{~cm}$ soil layer in China during the study period, and the spatial variation and vertical variation of these trends were also studied. (2) The coefficient of variation of the soil moisture of each layer of soil pixels during the study period was calculated, and $C_{v}$ of the spatial distribution of the soil moisture and the temporal and spatial fluctuations of the changes in soil moisture at different depths throughout China were analyzed. (3) The Hurst index was used to analyze the possible future trends of soil moisture in China. (4) The study time was for a total of 71 years from 1948 to 2018, longer than in previous studies. (5) The thickness of the studied soil layer was $0-200 \mathrm{~cm}$, greater than in previous studies. (6) The relationship between soil moisture and soil temperature was analyzed.

The paper is organized as follows. The data and methods are described in Sect. 2. In Sect. 3, we present the results of our analysis. In Sect. 4, we present a conclusion and discussion.

\section{Materials and Methods}

\subsection{Data source}

The data used in this study were from the GLDAS-Noah (global land data assimilation system-Noah-simulated) v2.0 and v2.1 soil moisture data sets. GLDAS was developed jointly by the Goddard Space Flight Center of NASA and the National Center for Environmental Forecasting of the United States Oceanic and Atmospheric Administration, and we adopted the soil moisture product from the Noah model. The time series of the v2.0 data sets was from 1948 to 1999 and that of the v2.1 data sets was from 2000 to 2018 . There were no v2.1 data sets from 1948 to 1999 . All the data have a spatial resolution of $0.25^{\circ} \times 0.25^{\circ(13)}$ and the unit of soil moisture is $\mathrm{kg} \cdot \mathrm{m}^{-2}$. The soil moisture is expressed by the average soil moisture content, and four layers with depths of $0-10,10-40,40-100$, and $100-200 \mathrm{~cm}^{(14)}$ were used to analyze the temporal and spatial variation characteristics of the soil moisture in China from 1948 to 2018. Liu et al. ${ }^{(6)}$ and Deng et al. ${ }^{(10)}$ confirmed that the GLDAS data and the Noah land surface process model have a good correlation with the observation data and can well reflect the temporal and spatial evolution characteristics of soil moisture, and they have been widely used in the study of soil moisture in China. ${ }^{(15-18)}$ 


\section{$2.2 \quad M-K$ trend test}

In the trend analysis of time series, the $\mathrm{M}-\mathrm{K}$ trend test is a rank correlation test of the rank and time series of a series of observations. ${ }^{(19)}$ It was originally proposed by Mann and Kendall, and many scholars continue to apply the $\mathrm{M}-\mathrm{K}$ trend test to analyze the trends in time series of measurements such as precipitation, runoff, temperature, and water quality. ${ }^{(20)}$ It is not disturbed by a small number of outliers in the sample, nor does it follow a certain distribution, and it is easy to calculate. In recent years, this test has been used to examine the trends of soil moisture, and its applicability has been demonstrated. ${ }^{(21-23)}$

In the $\mathrm{M}-\mathrm{K}$ trend test, time series variables are subject to $n$ independent and identically distributed random variables $x_{t}(t=1,2, \ldots, n)$, where $t$ is the length of the time series. The $\mathrm{M}-\mathrm{K}$ trend test defines the statistical variable $S$ :

$$
S=\sum_{k=1}^{n-1} \sum_{j=k+1}^{n} \operatorname{sgn}\left(x_{j}-x_{k}\right),
$$

where $n$ is the total number of samples; for all $k, j \leq n$, and $k \neq j, x_{j}$ and $x_{k}$ are sample values at times $j$ and $k$, respectively, and their distributions are different.

$\operatorname{sgn}()$ is a symbolic function defined as

$$
\operatorname{sgn}\left(x_{j}-x_{k}\right)=\left\{\begin{array}{cc}
1, & x_{j}-x_{k}>0 \\
0, & x_{j}-x_{k}=0 \\
-1, & x_{j}-x_{k}<0
\end{array}\right.
$$

The statistical variable $S$ has a normal distribution with mean $E(S)=0$ and variance $\operatorname{var}(S)=$ $n(n-1)(2 n+5) / 18$. When $n>10$, the standard normal statistical variable is calculated as

$$
Z=\left\{\begin{array}{cc}
\frac{S-1}{\sqrt{\operatorname{var}(S)}}, & S>0 \\
0, & S=0 . \\
\frac{S+1}{\sqrt{\operatorname{var}(S)}}, & S<0
\end{array}\right.
$$

In the bilateral trend test over a period, if $Z>0$, the soil moisture has an increasing trend in this period; if $Z<0$, the sequence has a decreasing trend, and the larger the absolute value of $Z$, the more obvious the trend. For the significance level $\alpha, Z_{1-\alpha / 2}$ is evaluated. When $|Z|<Z_{1-\alpha / 2}$, the null hypothesis is accepted, that is, the increasing or decreasing trend of the soil moisture is not significant. Otherwise, the original hypothesis is rejected, i.e., there is a clear upward or downward trend in soil moisture. Values of $|Z|$ greater than or equal to $1.28,1.64$, and 2.32 represent significance with confidence levels of 90,95 , and 99\%, respectively. ${ }^{(24-26)}$ 


\subsection{Coefficient of variation}

The coefficient of variation, also known as the standard deviation coefficient, is commonly expressed as $C_{v}$. It is a statistic used to measure the degree of variation of each observation data and is the ratio of the standard deviation to the mean. To analyze the degree of variation of soil moisture in China in the past 71 years, the value of $C_{v}$ for each pixel in the $0-200 \mathrm{~cm}$ soil layer was calculated. The soil moisture values of a pixel from 1948 to 2018 constitute a time series, and the coefficient of variation is calculated as

$$
C_{v}=\frac{\sigma}{\bar{\mu}}
$$

where $\sigma$ is the standard deviation of the soil moisture data of the time series and $\bar{\mu}$ is the mean value of the soil moisture data of the time series. For spatial distribution data, the greater the value of $C_{v}$, the greater the difference between regions and the more uneven the data; the smaller the value of $C_{v}$, the smaller the difference between regions. For time series data, the greater the value of $C_{v}$, the greater the fluctuation of the data and the more unstable it is; the smaller the value of $C_{v}$, the more stable the data is.

\subsection{Hurst index analysis}

The Hurst index is a statistical method first proposed by Hurst, a British hydrologist. It is a variable-scale range analysis method (rescaled range analysis, referred to as $\mathrm{R} / \mathrm{S}$ analysis). Later, Mandelbrot and Wallis further supplemented this method and perfected it theoretically, and developed it into a fractal theory for studying time series. ${ }^{(27,28)}$ In recent years, it has been widely used in hydrology, economics, climatology, and other fields. ${ }^{(29-31)}$ The principle is as follows:

Define the time series of soil moisture as $\xi_{(t)}$, where $t=1,2,3, \ldots, n$. For any positive integer $\tau \geq 1$, the mean sequence is defined as

$$
\overline{\xi_{(\tau)}}=\frac{1}{\tau} \sum_{t=1}^{\tau} \xi_{(t)}(\tau=1,2, \ldots, n)
$$

The cumulative dispersion sequence is

$$
X_{(t, \tau)}=\sum_{t=1}^{\tau}\left(\xi_{(t)}-\overline{\xi_{(\tau)}}\right)
$$

The range sequence is

$$
R_{(\tau)}=\max X_{(t, \tau)}-\min X_{(t, \tau)}(1 \leq t \leq \tau ; \tau=1,2, \ldots, n) .
$$


The standard deviation sequence is

$$
S_{(\tau)}=\left[\frac{1}{\tau} \sum_{t=1}^{\tau}\left(\xi_{(t)}-\overline{\xi_{(\tau)}}\right)^{2}\right]^{\frac{1}{2}} .
$$

The Hurst index is calculated as

$$
\frac{R_{\tau}}{S_{\tau}}=(c \tau)^{H}
$$

Here, $H$ is the Hurst index and $c$ is a proportional parameter. By taking the logarithm on both sides of the above formula and using the least-squares method to fit the double logarithm coordinate system $\left(\operatorname{In}\left(R_{\tau} / S_{\tau}\right)\right.$, In $\left.\tau\right)$, the slope of the straight line is obtained as the Hurst index $H$. The value range of $H$ is $(0,1)$. According to the calculated $H$ value, the trend of the time series can be judged. Specifically, three situations are considered:

(1) when $0<H<0.5$, the time series has anti-persistence, that is, the future trend is opposite to the past trend, and the closer $H$ is to 0 , the stronger the anti-persistence;

(2) when $H=0.5$, the time series is a random sequence, that is, the future trend is independent of the past trend;

(3) when $0.5<H<1$, the time series is a positive continuous series, that is, the future trend is consistent with the past trend, and the closer $H$ is to 1 , the stronger the sustainability.

\section{Results}

\subsection{Analysis of change trend of soil moisture over study period}

For soil moisture layers of $0-10,10-40,40-100$, and 100-200 $\mathrm{cm}$ in China, the $\mathrm{M}-\mathrm{K}$ trend test was used to calculate the $Z$ value of each pixel in the 71 years from 1948 to 2018, and the $Z$ value of each pixel was used to draw the trend distribution of soil moisture in each layer, as shown in Fig. 1.

It can be seen from Fig. 1 that the change trend of the soil moisture in the four layers has generally been consistent over the 71 years, that is, the soil moisture in most parts of the east has decreased, particularly in northeast China, central China, areas surrounding Yunnan Guizhou Plateau, and Taiwan Island, while the soil moisture in most parts of western China has increased, particularly in northern Xinjiang, western Inner Mongolia, and areas surrounding Tarim Basin. However, the $Z$ value in the southwestern part of the western Qinghai Tibet Plateau is less than 0 , which indicates that the soil moisture in this area has decreased.

With increasing soil layer depth, the decreasing trend of the soil moisture in northeast China becomes more obvious, especially in the deep soil in the west of northeast China and the northeast of Inner Mongolia Plateau. This is mainly because northeast China is an area with permafrost, ${ }^{(32)}$ where the melting of snow in spring increases the surface soil moisture, but the 

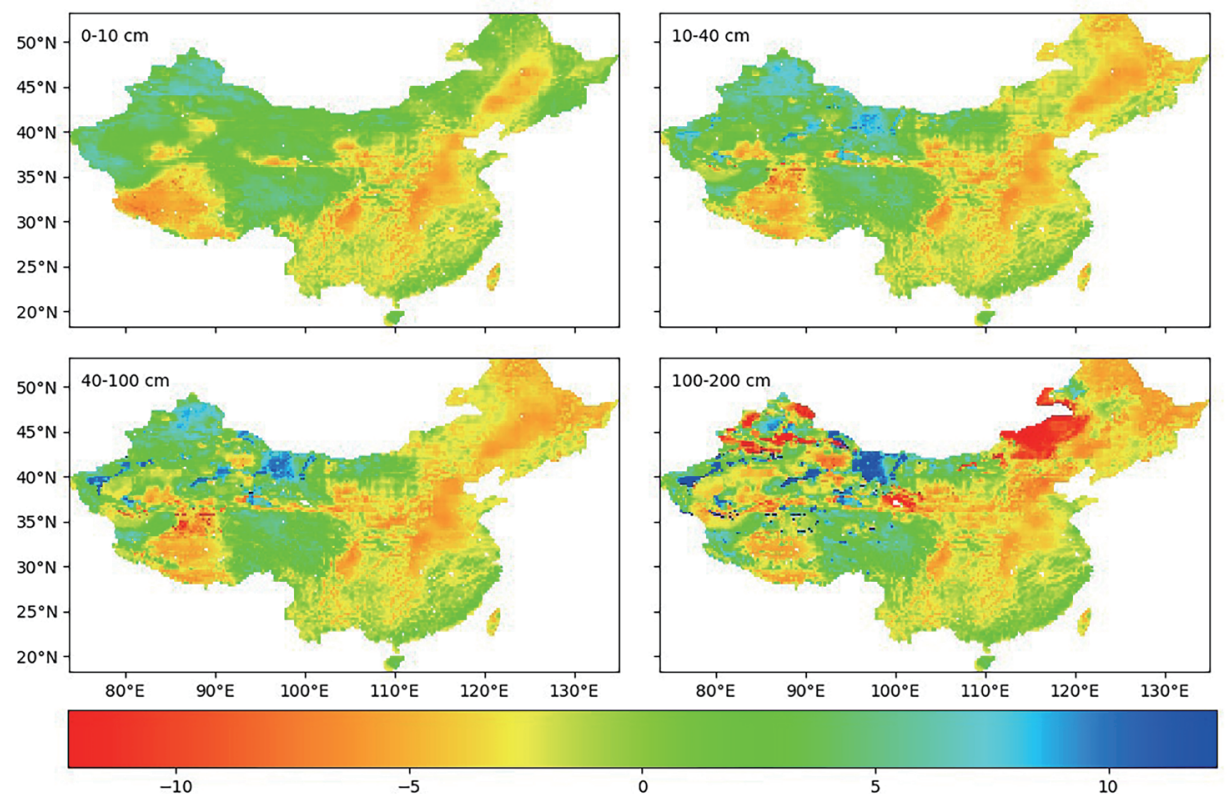

According to the vector map of GS (2016) 1570

Fig. 1. (Color online) Spatial-temporal trend of soil moisture in China from 1948 to 2018.

lower layer remains frozen and its moisture content does not increase. Moreover, precipitation plays a leading role in the change in soil moisture in northeast China, and the rainfall in the west of northeast China has decreased significantly in the past 71 years, resulting in a decrease in deep soil moisture. ${ }^{33,34)}$ In addition, in the Inner Mongolia Plateau, compared with the $100-200 \mathrm{~cm}$ soil layer, the $0-100 \mathrm{~cm}$ soil layer moisture is more closely related to the atmosphere and other influencing factors, and it is more vulnerable to periodic atmospheric changes, seasonal changes in radiation flux, and seasonal phases of the vegetation. Therefore, the periodic change in the surface soil moisture is more prevalent and the change in soil moisture is not obvious. ${ }^{(35)}$

According to our results, there are about 59200 pixels in each soil layer in the study area. In the $0-10 \mathrm{~cm}$ soil layer, there are 42258 pixels with $Z<0$ and 16942 pixels with $Z>0$; in the soil layer of $10-40 \mathrm{~cm}$, there are 41986 pixels with $Z<0$ and 17214 pixels with $Z>0$; in the 40-100 $\mathrm{cm}$ soil layer, there are 42470 pixels with $Z<0$ and 16730 pixels with $Z>0$; in the 100-200 cm soil layer, there are 42216 pixels with $Z<0$ and 16984 pixels with $Z<0$. It can be seen that the proportions of pixels with $Z<0$ and $Z>0$ are roughly the same in the four layers, with the pixels with $Z<0$ accounting for the majority ( $71.3 \%$ of pixels). This indicates that the change trend of the soil moisture in the four soil layers has basically been the same over the 71 years, with the soil moisture in most of China decreasing since 1948.

The confidence level is $99 \%$ when the absolute value of $Z$ is greater than or equal to 2.32 , $95 \%$ when the absolute value of $Z$ is greater than or equal to 1.64 and less than 2.32 , and $90 \%$ when the absolute value of $Z$ is greater than or equal to 1.28 and less than 1.64 . According to Table 1 , most of the pixels with a decreasing trend show a slightly decreasing trend. In the four 
Table 1

Distribution interval statistics of $Z$ value in soil layers.

\begin{tabular}{|c|c|c|c|c|c|c|c|c|c|}
\hline$Z$ value range & $\begin{array}{l}\text { Confidence } \\
\text { level }\end{array}$ & \multicolumn{2}{|c|}{$0-10 \mathrm{~cm}$} & \multicolumn{2}{|c|}{$10-40 \mathrm{~cm}$} & \multicolumn{2}{|c|}{$40-100 \mathrm{~cm}$} & \multicolumn{2}{|c|}{$100-200 \mathrm{~cm}$} \\
\hline$Z \leq-2.32$ & $99 \%$ & $12.4 \%$ & \multirow{4}{*}{$71.4 \%$} & $11.9 \%$ & \multirow{4}{*}{$70.9 \%$} & $12.5 \%$ & & $12.2 \%$ & \multirow{4}{*}{$71.3 \%$} \\
\hline$-2.32<Z \leq-1.64$ & $95 \%$ & $3.2 \%$ & & $3.0 \%$ & & $3.0 \%$ & $717 \%$ & $3.0 \%$ & \\
\hline$-1.64<Z \leq-1.28$ & $90 \%$ & $1.3 \%$ & & $1.4 \%$ & & $1.4 \%$ & $71.1 \%$ & $1.3 \%$ & \\
\hline$-1.28<Z<=0$ & $<90 \%$ & $54.6 \%$ & & $54.7 \%$ & & $54.8 \%$ & & $54.8 \%$ & \\
\hline $0<Z \leq 1.28$ & $<90 \%$ & $4.1 \%$ & \multirow{4}{*}{$28.6 \%$} & $4.3 \%$ & & $4.3 \%$ & & $4.1 \%$ & \multirow{4}{*}{$28.7 \%$} \\
\hline $1.28<Z \leq 1.64$ & $90 \%$ & $1.5 \%$ & & $1.5 \%$ & \multirow{3}{*}{$29.1 \%$} & $1.7 \%$ & 28,30 & $1.6 \%$ & \\
\hline $1.64<Z \leq 2.32$ & $95 \%$ & $3.7 \%$ & & $3.4 \%$ & & $3.6 \%$ & $28.3 \%$ & $3.4 \%$ & \\
\hline$Z>2.32$ & $99 \%$ & $19.3 \%$ & & $19.9 \%$ & & $18.7 \%$ & & $19.6 \%$ & \\
\hline
\end{tabular}

soil layers, an average of $54.7 \%$ of the pixels have $Z$ values greater than -1.28 and less than 0 , indicating that the area covered by these pixels has a downward trend with a probability of less than $90 \%$. However, most of the regions with a growth trend have stronger growth trend, and on average, the $Z$ value of $19.4 \%$ of the pixels in these four soil layers is greater than 2.32 , indicating that the areas covered by these pixels have a growth trend with a probability of $99 \%$. This shows that although the pixels in most regions of China show a decreasing trend, the decrease is small, and the confidence level of most pixels is less than 90\%. Combining Fig. 1 and Table 1 reveals that the variation trend of the soil moisture in the $0-200 \mathrm{~cm}$ soil layer in China has basically been the same in the past 71 years, the soil in most areas is gradually drying out, the trend is small, and most of these areas are distributed in the east of China. The decreasing trend is most prevalent in northeast China, central China, the areas surrounding Yunnan Guizhou Plateau, and Taiwan Island. In a few areas, soil moisture is gradually increasing, and these areas are mainly distributed in the western part of China, especially in the northern part of Xinjiang, the western part of Inner Mongolia, and the areas surrounding Tarim Basin.

\subsection{Coefficient of variation analysis}

By analyzing the spatial distribution of $C_{v}$ for the 71-year time series of soil moisture and the annual variation coefficient of soil moisture with time, we can find the fluctuation of each layer of soil moisture with space and time in the study area.

\subsubsection{Spatial distribution of time series $C_{v}$ of soil moisture}

We calculated $C_{v}$ of the time series of soil moisture values from 1948 to 2018 for each pixel in the $0-10,10-40,40-100$, and $100-200 \mathrm{~cm}$ layers as shown in Fig. 2. It can be seen that $C_{v}$ of the soil moisture values of each pixel in the four soil layers in the study area from 1948 to 2018 gradually increased from north to south, indicating that the degree of moisture dispersion in the soil in southern China was larger than that in the north up to a soil depth of $200 \mathrm{~cm}$. The greater the $C_{v}$, the more unstable the soil moisture in these regions has been in the past 71 years. This trend is most marked in the $0-10 \mathrm{~cm}$ soil layer. In this soil layer, $C_{v}$ is very large in the Sichuan Basin and to the south in south China, and in the region to the east of Wuyi Mountains, especially 

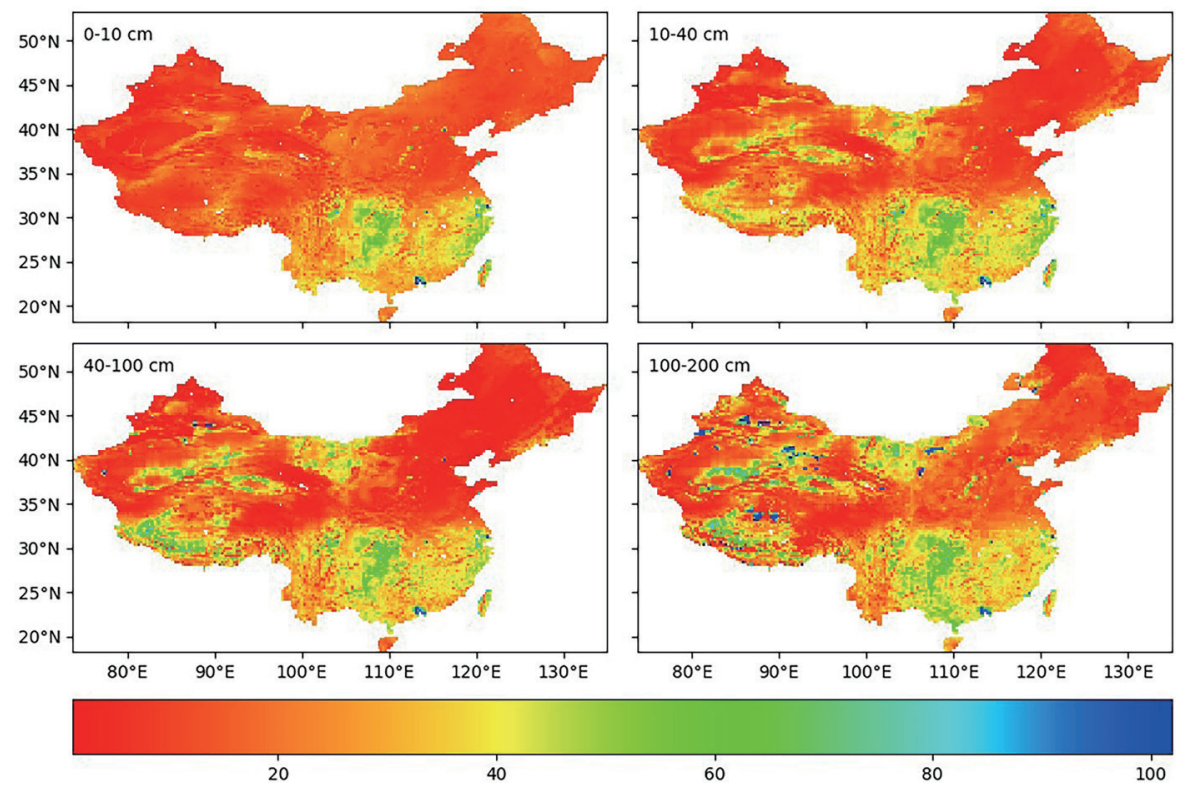

According to the vector map of GS (2016) 1570

Fig. 2. (Color online) Spatial distribution of time series $C_{v}$ of soil moisture for each layer.

in the Pearl River Delta region, which indicates that the soil moisture in these regions has fluctuated greatly in the past 71 years.

With increasing soil depth, $C_{v}$ of soil moisture increased gradually in southern Junggar Basin, central and western Inner Mongolia Plateau, Altun Mountains, Kunlun Mountains and the surrounding areas, Qaidam Basin, and southern Qinghai Tibet Plateau, especially in northern and southern Qinghai Tibet Plateau, Western Tianshan Mountains, and southern Junggar Basin. This shows that in these areas, soil moisture fluctuation increased with the soil depth in the last 71 years. However, in northeast China, Loess Plateau, northern China Plain, eastern Qinghai Tibet Plateau, and northern and western Tarim Basin, $C_{v}$ remained small with increasing soil depth, indicating that the soil moisture in the 0-200 cm layer in these regions has remained relatively stable in the past 71 years.

In summary, the change in soil moisture in the $0-200 \mathrm{~cm}$ soil layer has been more severe in southern China than in northern China, and the change in soil moisture in most areas of northeast, north, and east China has been more stable, with shallow soil more stable than deep soil.

\subsubsection{Time fluctuation of spatial distribution of soil moisture}

$C_{v}$ of the spatial distribution of soil moisture in the soil layer of $0-200 \mathrm{~cm}$ in the study area from 1948 to 2018 was calculated, and $71 C_{v}$ values in each layer of soil were used to analyze the variation of $C_{v}$ of the soil moisture spatial distribution with time in each layer of soil. Figure 3 shows the annual spatial $C_{v}$ for the four layers, from which we can see the spatial variation of soil moisture from 1948 to 2018. 


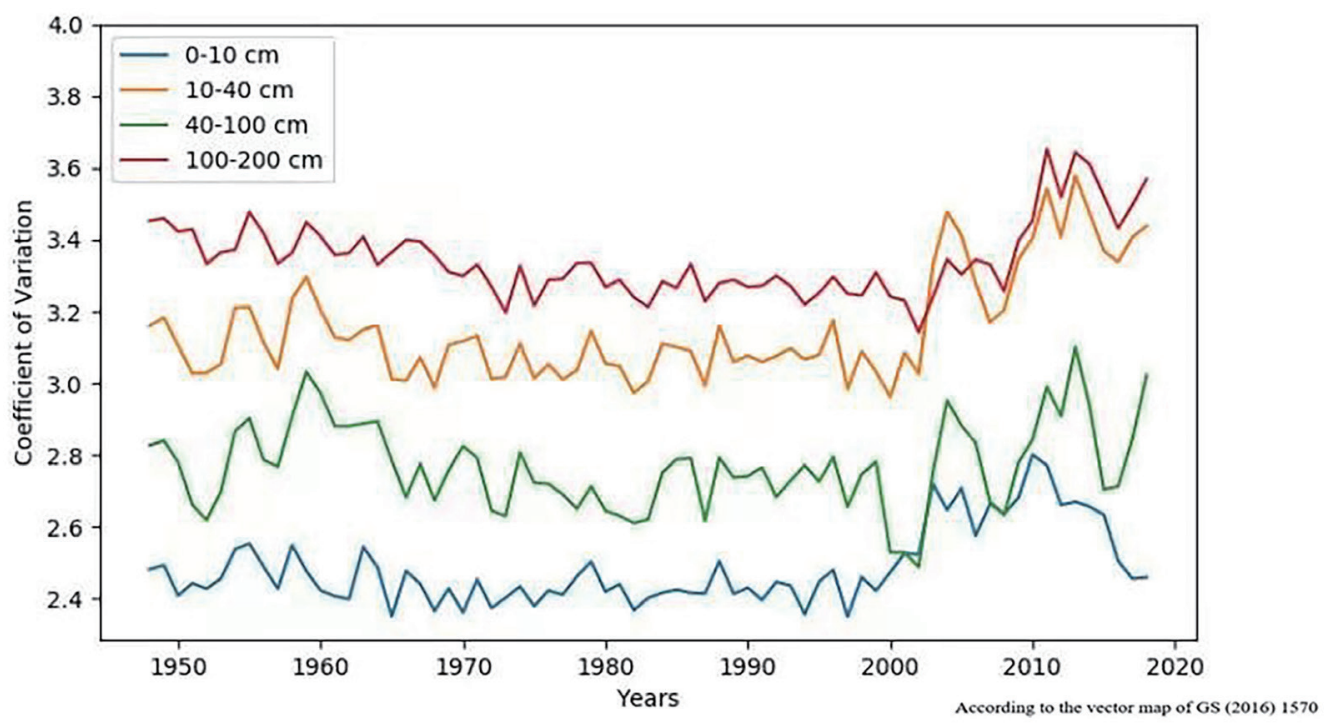

Fig. 3. (Color online) Time fluctuation of spatial distribution $C_{v}$ of soil moisture for each layer.

The blue, green, yellow, and red lines from bottom to top are the curves of $C_{v}$ for each layer of soil over the years with increasing soil depth, showing the variation of $C_{v}$ over the past 71 years. It can be seen that $C_{v}$ gradually increases with the soil depth, indicating that the spatial variation of soil moisture is less affected by surface factors and more affected by subsurface factors. In addition, the trend of $C_{v}$ in the four layers was roughly the same from 1948 to 2018, but the difference in moisture between the shallow soil regions is smaller than that in the deep soil regions and the variation trend of $C_{v}$ is basically the same. This fluctuation trend can be roughly divided into two stages.

First stage: 1948-2001. During this period, $C_{v}$ of soil moisture fluctuated and decreased gradually. It can be seen from Fig. 3 that the maximum values of $C_{v}$ in the $0-10,10-40,40-100$, and $100-200 \mathrm{~cm}$ soil layers are about $2.55,3.04,3.30$, and 3.46 and the minimum values are about $2.3,2.5,2.96$, and 3.13 , respectively. The difference between the maximum and minimum values for each layer is about 0.365 , which is smaller than that in the second stage. The results show that the humidity of the four soil layers in China is relatively uniform during this period, and the difference in soil moisture in the $0-200 \mathrm{~cm}$ soil layer between different parts of China is small. Although the difference has been fluctuating, it has decreased over time. Generally speaking, the difference in the humidity between the shallow soil regions is smaller than that between the deep soil regions.

Second stage: 2002-2018. After 2002, $C_{v}$ of soil moisture fluctuated greatly but on average tended to increase gradually. First, it increased sharply, reaching a large value around 2004, and then decreased to approximately the range of values before 2002. From 2008, $C_{v}$ began to increase significantly, reaching a maximum in 2011, which indicated that the soil moisture in the study area was extremely uneven between 2004 and 2011, and the difference between different parts of China was the largest. As a whole, the fluctuation range of $C_{v}$ of soil moisture in this stage was larger than that before 2002. $C_{v}$ of the $40-100 \mathrm{~cm}$ soil layer fluctuated the most, while 
$C_{v}$ of the $0-10$ and 100-200 cm soil layers fluctuated less but more than it did before 2002 . Indeed, $C_{v}$ of all four soil layers fluctuated more than it did before 2002. The results show that the soil moisture in the four layers was very uneven in China during this period, and the difference in soil moisture among different parts of China was large. Although the difference was not stable, it has been increasing, and the difference in shallow soil moisture among different parts of China is less than that in deep soil moisture.

In summary, $C_{v}$ of soil moisture in the study area fluctuated steadily from 1948 to 2001 while gradually decreasing. From 2002, the soil moisture in China became uneven, and the difference between different parts of China increased.

\subsection{Analysis of future trend of soil moisture}

The future trend of soil moisture at depths of $0-200 \mathrm{~cm}$ in China was analyzed on the basis of the Hurst index. The Hurst index values of each pixel of the 0-10, 10-40, 40-100, and 100-200 $\mathrm{cm}$ layers from 1948 to 2018 were calculated to analyze the future spatial trend of soil moisture in China. Figure 4 shows the Hurst index distribution maps of the four soil layers based on the Hurst index value of each pixel, which are used to analyze the future trend of moisture in each soil layer.

It can be seen that the Hurst index values of the four soil layers are basically the same, and the Hurst index values of the pixels in most of the study area are greater than 0.5 . This indicates that in the future, the moisture of the soil up to a depth of $200 \mathrm{~cm}$ in most areas of China will continue to change in the same way as it did from 1948 to 2018 as shown in Fig. 1, that is, the soil
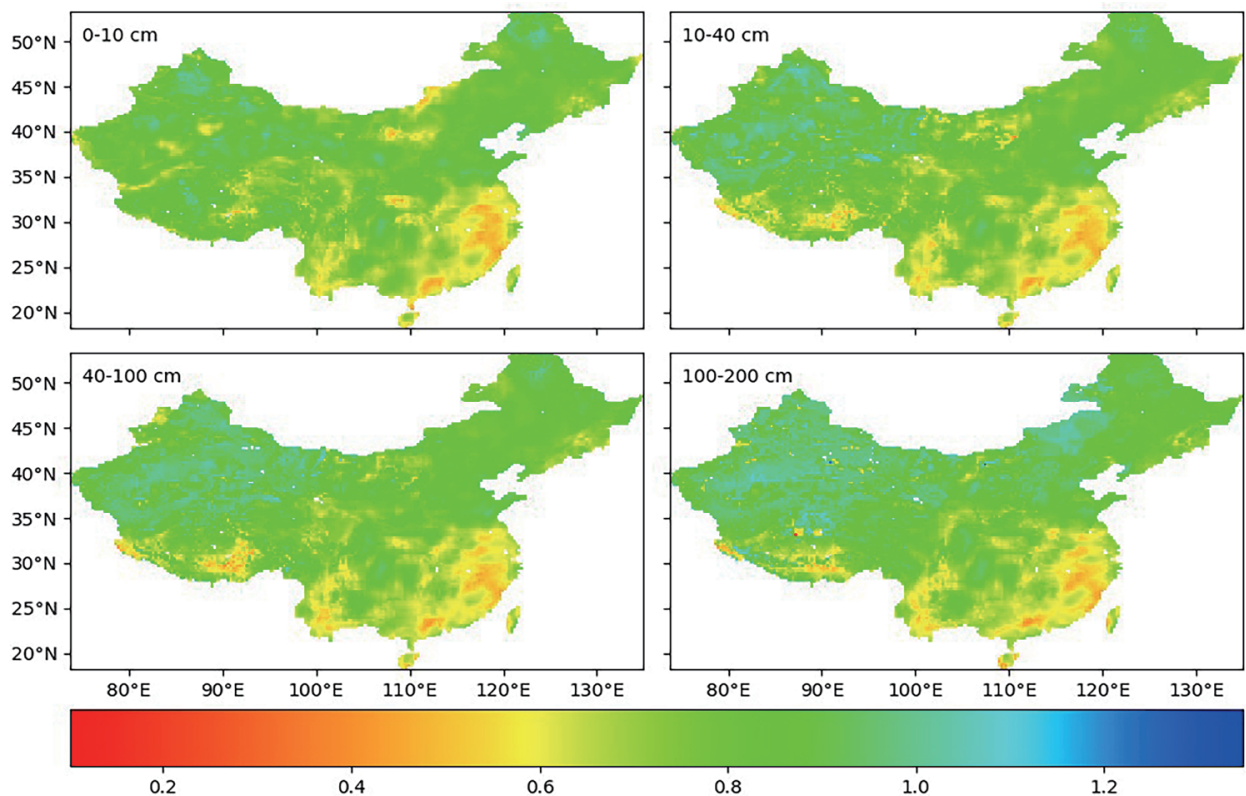

According to the vector map of GS (2016) 1570

Fig. 4. (Color online) Spatial distribution of Hurst index from 1948 to 2018 in each soil layer. 
in most areas will continue to dry. In particular, in some regions of Xinjiang, eastern Inner Mongolia, and northern Tibet, the Hurst index values are close to 1, so the trend of the soil moisture in these regions has strong continuity. For example, according to Fig. 1, the soil moisture of $0-100 \mathrm{~cm}$ in some areas of Xinjiang has a strongly increasing growth trend from 1948 to 2018. Combined with the fact that the Hurst index of some parts of Xinjiang is close to 1, it can be concluded that in the future, the moisture of the $0-100 \mathrm{~cm}$ soil layer in some parts of Xinjiang will continue to increase, and the growth rate will be higher than that in other parts of China where the moisture is increasing. The Hurst index of pixels in a small part of the area is less than 0.5 , which is mainly concentrated in southeast China, such as Wuyi Mountains, the plain of the middle and lower reaches of the Yangtze River, some areas of Yunnan Guizhou Plateau, and the western and eastern Himalayas, especially the Lingnan area. This shows that in the future, the soil moisture at depths of $0-200 \mathrm{~cm}$ in these areas will show the opposite trend to that from 1948 to 2018.

\subsection{Relationship between soil moisture and soil temperature}

In the time series studied, the main environmental factors affecting soil moisture are temperature, precipitation, solar radiation, and water vapor pressure. However, the main factors influencing the soil moisture variation in the soil layers of different depths in different years are different, which may be due to the joint effect of the uneven distribution of rainfall and the different contribution of soil moisture to evapotranspiration. However, soil temperature is the main factor affecting the variation of soil moisture at different depths. Soil temperature affects the redistribution of soil moisture by affecting the viscosity and surface tension of soil moisture. ${ }^{(36)}$ The research of Shuyan et al. ${ }^{(37)}$ on the factors influencing soil moisture shows that soil moisture is closely related to the change in temperature, and the correlation coefficients pass the significance test of $a=0.01$.

Combining our results with those of Zang et al. ${ }^{(38)}$ for the temporal and spatial variation of soil temperature in China from 1948 to 2018, we found that there is a clear inverse correlation between soil temperature and soil moisture in northeast China, central China, central and northern Inner Mongolia, Qinghai Tibet Plateau, and Taiwan Island, that is, soil moisture decreases with increasing temperature. This is mainly due to the increase in soil temperature, which accelerates the melting of ice in shallow soil, delays the freezing, shortens the period of frozen soil, and reduces the thickness of the frozen layer, which increase the evapotranspiration of soil water and reduce soil flow, resulting in a decrease in soil moisture content. ${ }^{(39)}$

\section{Discussion}

On the basis of a new research perspective, we analyzed the spatial and temporal variation of soil moisture in soil up to a depth of $200 \mathrm{~cm}$ in China in the last 71 years using the v2.0 and v2.1 data sets of the GLDAS-Noah model. The research results in this paper are basically consistent with previous research results. For example, they are consistent with the conclusion of Zhang ${ }^{(40)}$ and Chen ${ }^{(41)}$ that soil moisture is showing a decreasing trend on the whole, the conclusion of 
Cheng et al. ${ }^{(42)}$ that the deep soil in northeast China and some parts of Mongolia has become significantly drier than the shallow soil, and the conclusion of Deng ${ }^{(14)}$ that the soil will continue to dry in the future.

Although this paper studies the spatial-temporal variation trend and fluctuation of soil moisture in China in the past 71 years and the future trend of soil moisture, and discusses the relationship between soil moisture and soil temperature, there is a lack of analysis of the correlation with other important factors, such as soil texture, vegetation, topography, and human activities. ${ }^{(43)}$ The interaction between soil moisture and the Earth's system has been the subject of much recent research. Moreover, because soil drying is driven primarily by increasing soil temperature, processes that can affect the soil temperature will be able to mitigate or enhance soil drying, and such processes have become one of the hottest topics in the study of climate change.

\section{Conclusion}

On the basis of the analysis of the temporal and spatial variation of soil moisture in the $0-200$ cm soil layer from 1948 to 2018 in China, the following conclusions can be drawn:

(1) The trend in soil moisture in the four soil layers of 0-10, 10-40, 40-100, and 100-200 cm was basically the same over the 71 years. The soil moisture in most areas of eastern China showed a decreasing trend, with the soil drying over time in northeast China, central China, areas surrounding Yunnan Guizhou Plateau, and Taiwan Island. Except in the southwest of Qinghai Tibet Plateau, the soil moisture in most of western China is increasing. The change in the soil moisture was similar up to a depth of $100 \mathrm{~cm}$ over the 71 years from 1948 to 2018, but with increasing soil layer depth, the decreasing trend of the soil moisture in northeast China becomes more obvious.

(2) The variation of soil moisture up to a depth of $200 \mathrm{~cm}$ in southern China has been more severe than that in northern China. The soil moisture in Pearl River Delta was the most unstable, while that in most of northeast, north, and east China was relatively stable, with the smallest fluctuation during the 71 years. In the north and south of Qinghai Tibet Plateau, the west of Tianshan Mountains, and the south of Zhungeer Basin, the moisture fluctuation of surface soil was smaller and more stable than that of deep soil.

(3) Over the 71 years, the spatial variability of soil moisture in China was relatively small, the fluctuation range was small, and the difference in soil moisture in 0-200 cm soil layer between different parts of China gradually decreased. After 2002, the soil moisture in China became uneven, the difference in soil moisture among different parts of China increased, and the fluctuation range also increased. Also, with increasing soil depth, the spatial variation of soil moisture became more uneven, which indicates that the spatial variation of soil moisture is less affected by surface factors and more affected by subsurface factors.

(4) In the future, the change in soil moisture up to a depth of $200 \mathrm{~cm}$ in most regions of China is expected to be similar to that from 1948 to 2018. For example, the soil moisture in some regions of Xinjiang, eastern Inner Mongolia, and northern Tibet will continue to increase, while the soil moisture in southeast China will continue to decrease. 
(5) The trend of the soil moisture in the study area is closely related to soil temperature, usually with an inverse correlation, that is, the soil moisture decreases with increasing soil temperature, particularly in northeast China, central China, central and northern Inner Mongolia, Qinghai Tibet Plateau, and Taiwan Island.

The data used in this research is global data. Although we only used the part of the data for China, this data has been demonstrated to have high applicability in research on other parts of the world. The employed research methods have also demonstrated their applicability in studying long-term natural events and have been applied in other parts of the world. ${ }^{(14,40)}$ Therefore, relevant research data and the research methods in this study can be used to analyze soil moisture in other parts of the world.

\section{Acknowledgments}

GLDAS data from http://grace.jpl.nasa.gov were used in the study, which were acquired from the Goddard Earth Sciences Data and Information Services Center.

\section{Authors' Contribution}

Mengqing Geng designed the data processing and drafted the work. Dr. Feng Zhang and Dr. Xiaoyan Chang selected the topic, guided the overall thinking, reviewed the paper, and contributed to this work equally. Prof. Qiulan Wu and Lin Liang reviewed the paper and put forward suggestions for revision.

\section{References}

W. Zhang and R. Shen: Ground Hydrology and Groundwater Regulation (China Water and Power Press, 1998).

X. Zhang, X. Wu, and J. He: Acta Meteorol. Sinica 1 (2004) 310.

3 Y. Zhang, S. Zhang, W. Kong, L. Yang, and M. Wang: J. Lanzhou Univ. Sci. 55 (2019) 312.

L. Zhang, H. Lv, L. Wang, and B. Yang: Acta Geogr. 71 (2016) 314.

W. Zhang, T. Zhou, and C. Yu: Atmos. Sci. 3 (2008). 316.

6 L. Liu, D. Wei, X. Wang, and S. Cheng: Arid Meteorol. 37 (2019) 318.

7 C. Shi, Z. Xie, and H. Qian: Sci. Chin. Earth Sci. 54 (2011) 9.

8 L. Chen, J. He, and Y. Tan: J. Meteorol. Sci. 6 (2015) 35.

9 Q. Liu, J. Du, and J. Shi: Sci. Chin. Earth Sci. 56 (2013) 12.

10 M. Deng, X. Meng, Y. Ma, and Y. An: J. Arid Meteorol. 36 (2018) 320.

11 X. Sun, G. Fan, Y. Zhang, and X. Lai: J. Arid Meteorol. 2 (2019) 37.

12 H. Kim, R. Parinussa, A. Konings, W. Wagner, M. Cosh, V. Lakshmi, M. Zohaiband, and M. Choi: Remote Sens. Environ. 204 (2018) 260.

13 M. Rodell, P. Houser, U. Jambor, and J. Gottschalck: Bull. Am. Meteorol. Soc. 85 (2004) 3.

14 Y. Deng, S. Wang, X. Bai, G. Luo, L. Wu, Y. Cao, H. Li, C. Li, Y. Yang, Z. Hu, and S. Tian: Ecol. Indic. 110 (2020) 1 .

15 X. Li: Spatial-temporal Variability of Soil Moisture and Influencing Factors in Northwest Arid Are of China, Ph.D. Thesis, University of Chinese Academy of Sciences 321 (2019) 322.

16 Q. Sun: GRACE and GLDAS Data-based Estimation of Spatial Variations in Terrestrial Water Variations over Xinjiang, Ph.D. Thesis, Xinjiang University 323 (2015) 324.

17 P. Wu, Y. Feng, S. Liang, Q. Wu, and L. Wan: J. Beijing Norm. Univ. Sci. 52 (2016) 10.

18 K. Wang, X. Jin, R. Guo, S. Wei, and H. Liang: Geoscience 327 (2016). 328.

19 L. Zhang, S. Song, B. hao, and Y. Hou: J. Northwest A F Univ. Sci. Ed. 38 (2010) 330.

20 F. Pu: Environ. Sci. Surv. 331 (2014) 332. 
1 J. Zhang, X. Jin, X. Zhang, and X. Zhu: Hydrogeol. Eng. Geol. 91 (2019) 334.

22 H. Pan, G. Zhu, Y. Zhang, H. Guo, L. Yong, Q. Wan, H. Ma, and S. Li: Acta Geogr. Sinica. 74 (2019) 336.

23 M. Shen, Q. Zhu, Y. Wang, X. Mei, Q. Gou, and Y. Liu: J. Beijing For. Univ. 40 (2018) 338.

24 R. Lal: Science 304 (2004) 339.

25 M. Nashwan, S. Shahid, and N. Rahim: Theor. Appl. Climatol. 340 (2018) 341.

26 J. Liu, S. Fang, Q. Feng, Z. Wu, L. Han, J. Huang, and X. Bai: China Environ. Sci. 35 (2015) 343.

27 R. John, J. Chen, N. Lu, K. Guo, C. Liang, Y. Wei, A. Noormets, K. Ma, and X. Han: Remote. Sens. Environ. 112 (2008) 345.

28 X. Feng, Z. Feng, L. Luo, L. Qiu, and P. Liu: Arid Land Geogr. 31 (2008) 347.

29 R. Zamani, R. Mirabbasi, S. Abdollahi, and D. Jhajharia: Theor. Appl. Climatol. 129 (2016) 349.

30 Y. Liu, G. Xu, and P. Li: Res. Soil Water Conservation. 24 (2017) 99.

31 Z. Bai, L. Xue, and C. Zhang: Res. Soil Water Conserv. 26 (2019) 353.

32 A. Bo: Snow depth in Northeast China from 1980 to 2012 Effect on Soil Moisture, Ph.D. Thesis, Harbin Normal University (2019).

33 X. Zhang: Analysis and Assessment of Soil Moisture in China Based on the Situ Observation Data, Ph.D. Thesis, China University of Geosciences (Beijing) (2009).

34 Q. Sun: The Spatial-temporal Variation and Prediction Method of Soil Moisture in Northeast China, Ph.D. Thesis, Chinese Academy of Meteorological Sciences (2013).

35 Y. Chang: Characteristics of Soil Moisture Variation on Different Slopes in the Southern Margin of Inner Mongolia Desert Steppe, Ph.D. Thesis, Inner Mongolia Normal University (2021).

36 Y. Li, L. Shi, M. Xu, and W. Li: Arid Zone Res. 36 (2019) 355.

37 S. Li, H. Chen, W. Fang, and G. Zhao: Agric. Res. Arid Areas 25 (2007) 357.

38 M. Zang, M. Geng, F. Zhang, Q. Wu, and Y. Liang: Environ. Prot. 11 (2020) 359.

39 P. He: Research on Spatiotemporal Change and the Influence Factors of Vegetational Returning Green Stage in Northwest Mountainous Area of China Based on MODIS Data, Ph.D. Thesis, Yunnan Normal University (2018).

40 R. Zhang, L. Li, and Z. Zhang: Chin. J. Nat. 38 (2016) 5.

41 X. Chen, Y. Su, J. Liao, J. Shang, and L. Liu: J. Geophys. Res.: Atmos. 121 (2016) 1.

42 S. Cheng, X. Guan, and J. Huang: J. Geophys. Res.: Atmos. 120 (2015) 17.

43 Y. Pan and X. Wang: Hydrol. Process. 23 (2009) 11.

\section{About the Authors}

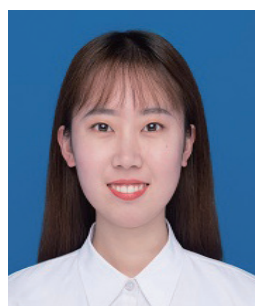

Mengqing Geng is currently an undergraduate studying surveying and mapping engineering in Shandong Agricultural University, China. (17686260738@163.com)

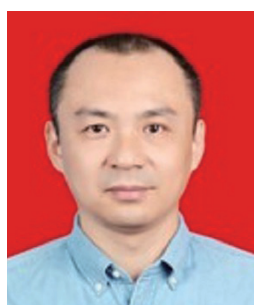

Feng Zhang received his B.S. degree from Shandong Normal University, China, in 2001, his M.S. degree from Ocean University, China, in 2008, and his Ph.D. degree from Shandong Normal University in 2015. Since 2017, he has been an associate professor at Shandong Agricultural University, China. His research interests are in the theory and application of 3S technology. (zhangfeng@sdau.edu.cn) 


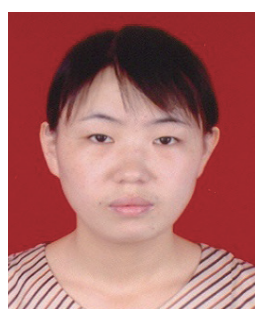

Xiaoyan Chang received her B.S., M.S., and Ph.D. degrees from Shandong Agricultural University, China, in 2002, 2005, and 2019, respectively. Since 2005, she has been a lecturer at Shandong Agricultural University. Her research interests are in landscape ecology and ecological risk assessment. (xychang@sdau.edu.cn)

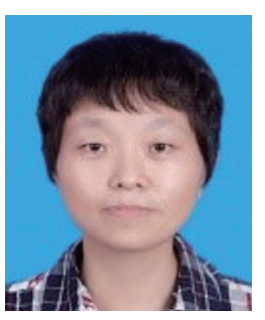

Qiulan Wu received her B.S. degree from Shandong University of Technology, China, in 1999, her M.S. degree from Shandong University, China, in 2001, and her Ph.D. degree from Shandong Agricultural University, China, in 2017. From 2002 to 2009, she was a lecturer, from 2010 to 2017, she was an assistant professor, and since 2018, she has been a professor at Shandong Agricultural University. Her research interests are in agricultural informatization and digital countryside. (zxylsg@sdau.edu.cn)

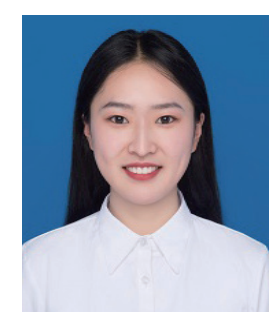

Lin Liang is currently an undergraduate studying surveying and mapping engineering in Shandong Agricultural University, China.

(lianglin_2001@163.com) 